\title{
44567 - ACUTE ANEMIA ACCENTUATES CEREBRAL INJURY FOLLOWING NEUROTRAUMA IN RATS
}

\author{
Gregory Hare, Department of Anesthesia, University of Toronto, Toronto, ON, Canada; \\ James Hutchison, Depts of Crit Care Med, The Hospital for Sick Children; \\ $C$ David Mazer, Department of Anesthesia, University of Toronto; \\ AT McLaren, Dept of Anesthesia, University of Toronto; \\ E Liu, Dept of Anesthesia, University of Toronto; \\ AJ Baker, Dept of Anesthesia, University of Toronto;
}

INTRODUCTION: A recent analysis of transfusion thresholds in patients suffering from neurotrauma suggests that these patients may benefit from a higher hemoglobin level. However, acute anemia has not been established as a clear risk factor for secondary brain injury following neurotrauma. We hypothesized that acute hemodilutional anemia will accentuate hypoxic cerebral tissue injury following unilateral traumatic brain injury (TBI) in a rat model of neurotrauma.

METHODS: With Animal Care Committee approval, isoflurane anesthetized rats underwent tail artery and vein cannulation to monitor mean arterial blood pressure (MAP), to measure arterial blood gases (ABG) and hemoglobin concentration (Co-oximetry) and to perform hemodilution. Anesthetized rats underwent standardized unilateral TBI or sham injury (n\&\#8805;8). Following injury, acute hemodilutional anemia was induced by exchanging $30 \mathrm{ml} . \mathrm{kg}-1$ of blood with an equal volume of pentastarch. Regional cerebral cortical blood flow (rCBF, laser Doppler) and caudate tissue oxygen tension ( $\mathrm{PBrO} 2$, Licox) were measured. In a separate group of rats $(\mathrm{n}=6)$, the effects of TBI and hemodilution on jugular venous oxygen saturation was assessed. Finally, cerebral contusion area and TUNEL positive cell counts were assessed in a third group of rats which were exposed to TBI alone or TBI and hemodilutional anemia $(n=3)$. Data (mean \pm SD) was analyzed by ANOVA or paired t-test.

RESULTS: Hemodilution reduced the hemoglobin concentration from $137 \pm 20$ to $52 \pm 13$ g.L1. No differences in ABG values were observed between groups. Following TBI, the baseline $\mathrm{PBrO} 2$ was decreased in the injured hemisphere $(12.2 \pm 1.6 \mathrm{mmHg})$ relative to uninjured controls $(18.2 \pm 5.2 \mathrm{mmHg})$. Hemodilution caused a further reduction in $\mathrm{PBrO} 2$ in the injured hemisphere $(7.6 \pm 2.3 \mathrm{mmHg}, \mathrm{p}<0.001)$, but not in the contralateral cerebral hemisphere. Bilateral rCBF increased comparably after hemodilution in both groups while the jugular venous oxygen saturation $(\mathrm{SjvO} 2)$ was significantly higher in the TBI group following hemodilution $(\mathrm{p}<0.001)$. The cerebral contusion area was significantly larger in the rats exposed to TBI and anemia $(7.6 \pm 5.4 \mathrm{~mm} 2)$, relative to those receiving TBI alone $(2.8 \pm 1.4 \mathrm{~mm} 2, \mathrm{p}<0.05)$.

Similarly, TUNEL positive cell counts were increased following TBI and anemia, relative to TBI alone ( $886 \pm 719$ vs. $568 \pm 406$ cells per coronal section, $\mathrm{p}<0.05$ ).

DISCUSSION: Severe hemodilutional anemia reduced the $\mathrm{PBrO} 2$ in the injured cerebral hemisphere following TBI. This occurred in association with an increase in $\mathrm{SjvO}$, relative to controls, suggested that TBI caused impairment of cerebral oxygen extraction. Increased contusion area and TUNEL positive cell counts following TBI and anemia supported the 
hypothesis that acute anemia accentuated hypoxic cerebral injury after neurotrauma. These data suggest that acute hemodilutional anemia may be an independent risk factor for secondary cerebral injury following acute neurotrauma. 